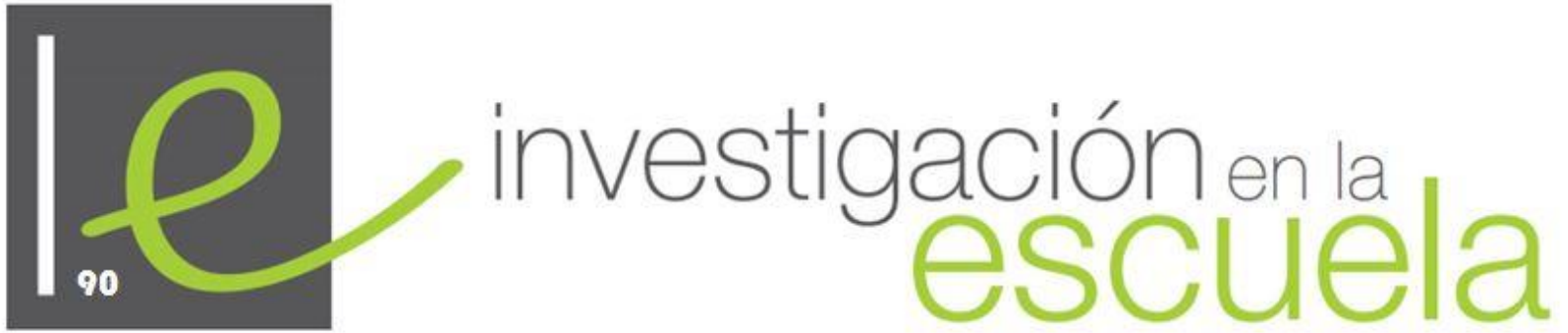

Revista académica evaluada por pares y de acceso abierto

\title{
El patrimonio inmaterial como estrategia educativa para fortalecer la identidad cultural en los jóvenes. Una intervención didáctica a través de la Historia Regional. Jonathan Almanza Ferrer \\ Escuela Técnica Superior de Turismo de Tuno (Campus Marseille) \\ Francia
}

Citación: Almanza, J. (2016). El patrimonio inmaterial como estrategia educativa para fortalecer la identidad cultural en los jóvenes. Una intervención didáctica a través de la Historia Regional. Investigación en la Escuela, 90, 19-33. Recuperado de: http://www.investigacionenlaescuela.es/ articulos/R90/R90-2.pdf

Resumen: Desde 2006, la política de Educación Patrimonial en México ha estado dirigida al desarrollo de la sensibilización cultural en los alumnos de Educación Media Superior mediante la asignatura de Historia Regional; sin embargo, tras haber identificado que las estrategias utilizadas en el plan de estudios consideran como recurso didáctico únicamente la herencia material y que ésta puede, como en el presente estudio de caso, mostrarse ajena a la cotidianeidad del alumno, se propone incluir el diseño de una intervención didáctica que se sirva del Patrimonio Inmaterial para contribuir al refortalecimiento de la Identidad Cultural.

Palabras clave: "Educación Patrimonial"; "sensibilización cultural"; "conciencia histórica";

"Identidad Cultural"; "Patrimonio Inmaterial"; "intervención didáctica"; "modelo de competencias"; "Historia Regional".

The intangible heritage as a teaching strategy for the cultural identity awareness in young people. A didactic intervention through the Regional History.

Abstract: Since 2006, heritage education policy in Mexico has tried to develop cultural consciousness in High School students through the course entitled Regional History. However, after 
identifying that the strategies proposed in the course consider only material heritage which sometimes can be composed, like in this investigation, by goods outside the daily lives of school students, this work designs an educational intervention with strategies based on an intangible heritage in order to contribute to the development of cultural identity.

Key words: "Heritage education"; "cultural consciousness"; "historical consciousness"; "cultural identity"; "intangible heritage"; "didactic intervention"; "competency model”; "regional history".

\section{Le Patrimoine Immatériel, une stratégie pédagogique pour sensibiliser les jeunes à leur culture.}

Resumè: Depuis 2006, la politique d'éducation au patrimoine au Mexique a été visée pour développer la sensibilisation culturelle aux lycéens à travers la matière d'Histoire Régionale ; pourtant, après avoir identifié que les stratégies utilisées au plan d'études ne considèrent que le patrimoine matériel, lequel peut constituer un élément étranger, comme dans le présent travail, pour le quotidien des étudiants; ce travail propose le design d'une intervention didactique qui s'en serve du patrimoine immatériel à fin de contribuer au développement du renforcement de l'identité culturelle.

Mosts clé: "Éducation au patrimoine"; "sensibilisation culturelle"; "conscience historique"; "identité culturelle"; "patrimoine immatériel"; "intervention didactique"; "modèle de compétences"; "histoire régionale".

\section{Introducción}

El patrimonio cultural es una dote sensible al tiempo, a los estragos de la intemperie, al trato agresivo e irrespetuoso, pero, sobre todo, al olvido; es por ello que en aras de su resguardo y continuidad se ha visto en la educación un potencial para afianzar los vínculos que unen al individuo con el grupo social y así fortalecer su Identidad Cultural.

La Educación Patrimonial emprende acciones con el fin de desarrollar en las nuevas generaciones una sensibilización a los componentes culturales que le han sido legados y que constituyen parte de su identidad como sujeto. Sin embargo, la cotidianeidad actual evidencia la persistencia de actitudes de desapego e indiferencia en los jóvenes hacia estos elementos; situación que vuelve necesaria la reflexión respecto al impacto de dichos esfuerzos educativos.

En México, la tendencia detectada por Vizcaíno (2005) gracias a los datos estadísticos aportados por el World Value Survey (WVS), mostraba ya cómo las identidades locales presentaban una tendencia al detrimento, mientras que la auto-adscripción al mundo como un todo se manifestaba en ascenso; tendencia que ve su continuidad en el último reporte realizado en el marco del programa 2010-2014 del WVS, el cual revela que en las generaciones más jóvenes se tiende mayormente a rechazar la idea de formar parte de una comunidad local, frente a una ubicación como ciudadano del mundo (Moreno, 2012); dejando en claro entonces cómo la sociedad mexicana envuelta en este contexto globalizador es fuertemente impactada por los modelos culturales cosmopolitas promovidos internacionalmente.

En el siguiente trabajo se muestra en un primer punto la política educativa que México ha emprendido en el Nivel Medio Superior para fortalecer la Identidad Cultural, y se abordan los conceptos derivados de la Educación Patrimonial y el binomio Patrimonio-Identidad. Posteriormente se presenta, a manera de caso, la experiencia realizada en dos grupos de una escuela de Nivel Medio Superior ubicada en la periferia de Puebla, México, es decir, apartada de los bienes culturales materiales reconocidos institucionalmente. A través de un diseño cuasi-experimental se analizan las estrategias emprendidas durante un curso de Historia Regional con el fin de desarrollar 
en los alumnos la Identidad Cultural, identificando las causas que dificultan el alcance de dichos objetivos, y planteando una propuesta con fundamentación teórica que busque enfrentar el problema.

\section{La Educación Patrimonial a través de la Historia Regional}

A medida que el proceso de globalización ha ido ganando terreno en las últimas décadas, ha crecido paralelamente el deseo de revalorar, proteger y transmitir lo que se considera una herencia cultural que diferencia y forma parte de la construcción de una identidad, llevando incluso a la reconsideración del concepto de etnicidad que incluye las cuestiones "de dónde provengo, qué es lo que me hace ser lo que soy, en una palabra, la identidad natural” (Baumann, 2001, p.33).

Pese a que la familia es la unidad social que tradicionalmente es la responsable de trasmitir la herencia cultural a los miembros más jóvenes, el rol de la escuela como organismo social se torna en un colaborador que más que dar una continuidad a la trasmisión, favorece la revalorización de los símbolos de identidad no como objeto de culto sino como elementos propios que integran el entorno cotidiano en el que el alumno se desenvuelve. "Algunos estudiantes tienen el privilegio de vivir con sus familias experiencias que los ponen en contacto con las huellas dejadas por el pasado, de tal modo que pueden construirse un capital histórico; pero partiendo del hecho de que la mayoría de los niños no tiene esta suerte, es en el colegio donde deben vivir experiencias similares" (Loison, 2008, p.87).

Una de las características primordiales de la cultura es la transmisión para su permanencia, y es en esta función donde la escuela se ha convertido en una figura que, más que meramente colaborar, recibe la responsabilidad de favorecer la revalorización de los símbolos de identidad a través de la Educación Patrimonial, entendida ésta desde el punto de vista de la acción educativa para reconocer, afianzar y amparar, por su valor auto-asignado, los bienes colectivos de los que goza la generación actual (Fontal Merillas, 2003).

En México las consecuencias de una carencia de identidad local en los jóvenes son evidentes en actitudes como la negación del yo indígena, la individualidad, la preferencia por lo nuevo (que rápidamente se torna obsoleto) en contraposición a lo tradicional, y la predilección por lo foráneo en cuanto al consumo cultural, lo que incluye la asimilación de sus mensajes y un reconocimiento cultural (Bisbal, 2001).

Bajo este escenario, la Reforma Integral de la Educación Media Superior (RIEMS) propuesta por la Secretaría de Educación Pública de México (SEP) en el 2006, encomienda a la asignatura de Historia Regional proporcionar elementos que permitan al estudiante asumirse como protagonista del rescate, la conservación y/o fortalecimiento de las manifestaciones culturales de su localidad, es decir, fomentar una Identidad Cultural a través de la Conciencia Histórica.

\section{La Identidad Cultural y la Historia.}

El término identidad es un concepto polisémico, puesto que desde la filosofía, la psicología, la sociología o la antropología, puede abordarse con un marco de referencia muy diferente, en donde incluso "debido a la revolución tecnológica y a su repercusión en la creación de nuevas formas de socialización las definiciones de identidad individual y colectiva requerirían una nueva revisión" (Delors, 1996, p.61).

Sea la perspectiva que se elija, la connivencia en cuanto a la conceptualización del término radicará en la consideración de una incorporación satisfactoria del sujeto a un modo particular de significar la realidad. Desde una faceta cultural "la identidad es un aspecto crucial de la reproducción: es la cultura internalizada en sujetos, subjetivada, apropiada bajo la forma de una conciencia de sí en 
el contexto de un campo ilimitado de significaciones compartidas con otros" (Gorosito, 1998, p.102); por ello la identidad, vista desde una perspectiva cultural, posibilita abordar su carácter transmisor y correlativo en el binomio identidad-cultura.

Desde este enfoque cultural, el presente trabajo se apoya en el concepto de Identidad Cultural que plantea la Declaración de México en la Conferencia Mundial sobre Políticas Culturales de 1982, que la define como "la defensa de las tradiciones, la historia y los valores morales, espirituales y éticos heredados de las generaciones pasadas" (UNESCO, 1982).

Los principios que la Declaración enuncia como ejes rectores de las políticas encaminadas al desarrollo de la Identidad Cultural, dejan entrever cómo ésta se constituye por el Patrimonio Material e Inmaterial; de ahí que se torne indispensable en el ámbito educativo destacar el papel de la Historia en el desarrollo de la construcción de la identidad, pues el tiempo y espacio se vuelven referentes forzosos en el proceso que permite a cada individuo fundamentar un sentimiento de pertenencia respecto al grupo del que forma parte. Como Sánchez Quintanar comenta: "la asunción de mi identidad regional implica identificarme y diferenciarme de los sujetos que junto conmigo constituyen mi mundo regional... sólo que la identificación de cada individuo con sus semejantes habrá de construirse no sólo en un sentido horizontal (espacio), sino también en un sentido vertical, es decir, en el tiempo" (Sánchez Quintanar, 2002, p.54).

\section{El Patrimonio Cultural Inmaterial}

Aunque en un inicio la concepción del patrimonio cultural pretendía incluir todo tipo de manifestaciones sociales que representan un legado histórico, la vulnerabilidad que presentan hacia ciertos factores no impacta de igual modo a todas estas expresiones. Existen bienes que por sus aspiraciones originales de permanencia se muestran más sólidos, aunque no por ello menos sensibles ante el paso del tiempo; sin embargo, otros tantos por su carácter de inmaterialidad tienden a ser especialmente permeables a los cambios.

La UNESCO previendo tales diferencias, convocó la Convención para la Salvaguardia del Patrimonio Cultural Inmaterial en París; para la formalización de dicho término en su artículo segundo menciona la inclusión de "usos, representaciones, expresiones, conocimientos y técnicas junto con los instrumentos, objetos, artefactos y espacios culturales que les son inherentes- de las comunidades" (UNESCO, 2003). Dentro de las características de dicho legado cultural, se le reconoce su carácter evolutivo, representativo e integrador por su contribución a la cohesión social al fomentar un sentimiento de identidad y responsabilidad comunitaria.

De dicha definición se entiende que se considera este tipo de patrimonio como un crisol de la diversidad cultural que por un lado, al ser un factor clave en la construcción de la identidad, se vuelve un objeto de cuidado por su carácter de fragilidad; y, por otra parte, al ser un patrimonio vivo, reconoce su capacidad para estar en constante evolución. "La extraordinaria relevancia del Patrimonio Inmaterial es que, además de un bien colectivo abierto al goce y al disfrute, es un sistema colectivo que modela; educa y/o fomenta la imitación en el individuo y que interactúa con su capacidad de experimentar, crear e innovar" (Asiáin y Aznárez, 2012, p.46).

\section{El modelo simbólico-social en la Educación Patrimonial}

Una vez recalcada la importancia de la consideración del Patrimonio Inmaterial, paralelamente al Material, como estrategia para el fortalecimiento de la Identidad Cultural, el modelo simbólico-social de Educación Patrimonial planteado por Fontal Merillas (2003) permite integrar conceptual y metodológicamente las aspiraciones de la asignatura de Historia Regional. 
La misión del modelo consiste en auxiliarse del valor simbólico de los bienes culturales para, más allá de transmitirlos, favorecer en el sujeto el desarrollo de la identidad puesto que, como asegura Fontal Merillas (2003), la auto-adscripción a un grupo parte del reconocimiento de la importancia del patrimonio cultural en la construcción de procesos simbólicos que contribuyen a configurar la identificación del sujeto. Es decir, al pretender colaborar en la construcción de la identidad se enfocan las acciones en el desarrollo de la apropiación, la concientización, la valoración y la sensibilización hacia la herencia cultural, que propiciarán la salvaguarda del patrimonio.

La propuesta del modelo simbólico-social favorece, a partir de los elementos culturales, la memoria colectiva; es decir, mediante el conocimiento del pasado se comprenden las consecuencias de la acción presente en el futuro, permitiéndole al sujeto reafirmar, disfrutar, preservar y recrear la propia identidad. Sin embargo, se requiere poner especial cuidado en el trasfondo político que pudiese plantearse a través de él. Henríquez Sánchez advierte sobre los problemas de carácter ético en cuanto a las políticas encaminadas a la salvaguarda cultural y menciona que "el patrimonio o, mejor dicho, los criterios de selección del mismo, actúa como un potente mecanismo de control social" (Henríquez Sánchez, 2011, p.205).

La enorme posibilidad que ofrece para difundir ideales nacionalistas y etnocentristas se vuelve un riesgo que podría atentar contra las pretensiones de cohesión social, puesto que afirmar la identidad constituye un acto liberador que "si resulta mal interpretado, contribuye asimismo a hacer difíciles, cuando no imposibles, el encuentro y el diálogo con el otro" (Delors, 1996, p.61). Por ello, las propuestas deben consideran la ambivalencia en cuanto a la noción de otredad y el respeto intercultural.

\section{Estrategias fraccionadas en la Educación Patrimonial: el estudio de caso}

El modelo educativo en México se ha centrado en los últimos años en el desarrollo de competencias consideradas desde la perspectiva de la Asociación Nacional de Universidades e Instituciones de Educación Superior como "el conjunto de conocimientos, habilidades y destrezas, tanto específicas como transversales, que debe reunir un titulado para satisfacer plenamente las exigencias sociales" (De Allende y Morones, 2006, p.4).

La finalidad educativa de dicho modelo es influir en la capacidad del sujeto para responder al contexto actual, y en cuanto a Educación Patrimonial se refiere, se busca promover en el aula la competencia que plantea la sensibilización a las manifestaciones culturales, que como hecho histórico compartido permite desarrollar un sentido de identidad en la vida de su comunidad y región.

Sin embargo, ante el último reporte de Moreno (2012) en el marco del programa 2010-2014 del World Value Survey, donde evidencia cómo en las generaciones más jóvenes en México existe una tendencia al rechazo de la idea de una auto-adscripción local, se vuelve ineludible la reflexión respecto al porqué del poco impacto que se logra en los alumnos tras haber cursado una asignatura implementada ex profeso para el desarrollo de la Identidad Cultural.

Si bien "la apropiación de la historia es una labor compleja en la que se pretende comunicar cómo los objetos tienen valor no por lo que son sino por lo que representan" (Martín Guglielmino, 2007, p.201), la presente investigación buscó hacer una propuesta para justamente posibilitar una mejor comunicación de los valores entre sujeto y objeto.

Después del análisis del programa oficial de la materia de Historia Regional se conjeturó como primicia que la búsqueda del desarrollo de un sentido de identificación simbólica se plantea como consecuencia del acercamiento que el alumno tiene sólo al legado histórico que ha dejado una huella material, presuponiendo que las actitudes de apropiación y respeto se logran simplemente conociendo el bien patrimonial; es decir, la finalidad de las estrategias planteadas en el programas 
tienden a recaer en el modelo historicista planteado por Fontal Merillas (2003) lo cual presupone que la transmisión teórica es suficiente para promover actitudes en el sujeto.

De carácter cuantitativo, se procedió mediante un cuestionario mixto a obtener datos que constituyeran la base para generar estadísticas respecto a la conducta de la muestra no probabilística sobre la cual se basó el estudio: un diseño cuasi-experimental donde se seleccionó un grupo control y otro experimental conformado por estudiantes del primer año de Educación Media Superior con un número similar de participantes (30 alumnos).

La característica fundamental del instituto en el que se realizó el presente trabajo es que su localización corresponde a la periferia de Puebla, y ya que en esta ciudad el Patrimonio Cultural Material reconocido institucionalmente se encuentra centralizado, geográficamente hablando, la cuestión era probar si efectivamente una estrategia de sensibilización basada en lo meramente tangible podía provocar un impacto limitado.

Tras la aplicación de una prueba pre-test en ambos grupos se constató un carente sentido de auto-percepción como sujetos históricos, la tendencia a identificarse mayormente con los modelos globales promovidos por la publicidad y con los ideales primermundistas más que en los elementos culturales de la propia localidad, así como el poco interés por el acercamiento a su legado históricocultural, declinando así en el menoscabo de una conciencia de resguardo y preservación.

Como antecedente a dicha situación, Pinto y Molina (2015) ya habían puesto en evidencia en sus estudios en España y Portugal el poco eco de la Educación Patrimonial a través de la Historia a pesar de las reformas curriculares, e igualmente señalaron la falta de formación del profesorado para aproximar de manera más pertinente las fuentes patrimoniales. La pertinencia, por tanto, es un elemento clave en la educación, pues el contexto de los estudiantes, en el caso del presente estudio: su proximidad al legado material, debía considerarse para abrir un puente entre lo que históricamente les pertenece y lo que regularmente aprecian como elemento de identidad.

Una Educación Patrimonial que a pesar de estar dirigida a la concientización de la salvaguarda de la herencia cultural no ocupe estrategias vivenciales que contribuyan a una percepción de apropiación, degenerará en la apatía hacia los elementos culturales al ser éstos considerados por el alumno como símbolos ajenos a su realidad; por ello Gabardón (2005) plantea la necesidad de utilizar el patrimonio local como referente vivo más cercano al alumnado, que despierte la síntesis de valores que se identifica en un bien histórico.

El diseño de una estrategia fragmentada en lo referente a la enseñanza del patrimonio, que busca acercar y sensibilizar al alumno a su legado histórico sólo mediante lo material conlleva a valoriza bienes que incluso geográficamente se encuentran separados de la realidad del sujeto. Posteriormente a la revisión del curso de Historia Regional y el análisis de los datos obtenidos en el pre-test, se planteó el diseño de una estrategia que implementara también la enseñanza del Patrimonio Cultural Inmaterial.

El Patrimonio Inmaterial concibe una aproximación del individuo al legado cultural mediante el reconocimiento de las huellas que la historia ha dejado en su cotidianeidad como bienes legítimamente heredados, aceptados como propios y dignos de preservación. Este tipo de herencia puede ser considerada, de acuerdo con Gil-Díez (2013), como parte de un proceso de democratización y reivindicación de un bien patrimonial.

En contra del coleccionismo de la educación bancaria planteado por Freire (2005), las aspiraciones simbólico-sociales de la Educación Patrimonial incluyen una educación problematizadora que propone una horizontalidad, pues mediante este tipo de enseñanza se pretende librar al sujeto de la alienación de unos saberes arbitrariamente elegidos, que en el caso de la Educación Patrimonial repercuten en la desvinculación entre los conocimientos aprendidos: los bienes culturales y la cotidianeidad del individuo. 


\section{E1 diseño de la intervención didáctica a través del Patrimonio Inmaterial}

Bajo este contexto, se buscó implementar una estrategia de intervención didáctica para favorecer en el alumno del instituto en cuestión el desarrollo de la competencia genérica planteada en la RIEMS, que busca la sensibilización a las manifestaciones culturales, así como la participación en la apreciación y la interpretación de sus expresiones en los distintos géneros. Sirviéndose de la enseñanza del Patrimonio Inmaterial se pretendió complementar las estrategias de la herencia cultural material que el programa de la asignatura de Historia Regional plantea y que pudieran recaer en un enfoque historicista en la Educación Patrimonial.

Con el fin de describir el influjo de dicha intervención y medir el impacto de las estrategias se procedió a la aplicación en ambos grupos de una prueba pre-test y otra post-test que permitieran identificar los logros en cuanto al desarrollo de la Identidad Cultural, concepto considerado a partir de la definición de la UNESCO (1982); de tal manera que dichos resultados fueran susceptibles de ser interpretados y sirvieran para corroborar o rechazar la hipótesis planteada: si la implementación de un modelo novedoso de enseñanza del Patrimonio Inmaterial puede lograr el desarrollo de la Identidad Cultural.

La intervención fue de carácter constructivista, realizada durante nueve sesiones continuas, planteando como objetivo el desarrollo de competencias que contribuyeran a la sensibilización, la participación y la interpretación de las manifestaciones culturales del grupo al que se pertenece, planteando así que el estudiante del grupo experimental:

1. Reconociera el vínculo de unidad de la sociedad en la que vive mediante la elaboración y comparación de un listado de ingredientes para la preparación de un platillo típico de la gastronomía local, a fin de lograr una mayor sensibilización respecto al sentido de pertenencia a un grupo social.

2. Reconociera las técnicas de elaboración de las bebidas más típicas de la comunidad en la que vive, a través de un cuadro de relación, para apreciar su valor histórico.

3. Redescubriera los sonidos presentes en el cotidiano local por medio de una dramatización de éstos, con el fin de sensibilizar a los elementos de identidad.

4. Reconociera la diversidad musical presente en su localidad como un medio de expresión social, mediante la recomposición de una estrofa de una canción que sensibilizara respecto al significado de ser parte de la comunidad poblana.

5. Percibiera la influencia de las lenguas indígenas y de regionalismos en el español, enlistando los mexicanismos que usualmente ocupa en su vida diaria, para sensibilizarse respecto a la relación lengua-identidad.

6. Apreciara los saberes indígenas de su localidad, mediante la elaboración de un recetario de la medicina tradicional para sensibilizar respecto a la presencia indígena en su presente.

7. Tomara conciencia respecto al valor de las manifestaciones religiosas de su localidad, mediante un cuadro de doble entrada ilustrado donde se relacionaran los santos y las vírgenes de veneración actual de la propia comunidad, producto de la transculturación de la cosmovisión mesoamericana y el catolicismo español.

8. Apreciara la dificultad de los oficios tradicionales a través de la elaboración de unas figuras en papel picado para sensibilizar respecto al arte local

9. Reflexionara respecto a los shocks culturales que podría resentir una persona ajena a la comunidad durante una fiesta tradicional de la localidad, a través de una carta de recomendaciones respecto a las diferencias culturales, con el fin de valorar el sentido de pertenencia a la comunidad en la que vive. 
En estas propuestas educativas, se procuró una correspondencia directa entre el discente y el contenido, variables en el proceso educativo consideradas clave para el aprendizaje significativo donde "los contenidos tienen que engarzarse con los esquemas de conocimiento previos del alumno para adquirir sentido” (Fontal Merillas y Marín Cepeda, 2011), de tal manera que se tomó en consideración el bagaje cultural previo del sujeto para llegar a través de una estrategia deductiva a un conocimiento más general.

Durante la calendarización del tema Historia Regional, específicamente en el subtema de Poblamiento y agricultura en las sociedades Mesoamericanas, cuyo propósito es que el alumno delimite su región y reconozca los elementos culturales de su entorno, se implementó en el grupo experimental la intervención didáctica que buscó promover la competencia genérica de "es sensible a las expresiones culturales y participa en la apreciación e interpretación de sus expresiones en distintos géneros" (SEP, 2008:2). Las secuencias didácticas para las nueve sesiones que duró el ejercicio abarcaron el valorar los elementos de las civilizaciones del pasado que se integran al presente regional y que determinan e identifican al estudiante como sujeto socio histórico; analizar la presencia y relevancia de las diversas civilizaciones que habitaron el espacio regional; y examinar los diferentes aspectos de interacción en la región a la luz de los acontecimientos y etapas de la historia nacional.

Los alumnos, sirviéndose principalmente como estrategia de enseñanza de contenidos relativos a la lengua, la música, y los saberes comunitarios:

- Concluyeron la importante influencia mesoamericana tanto en la alimentación cotidiana así como en la verbalización de ideas y pensamientos; considerando el enriquecimiento cultural reflejado en la gastronomía y la lengua gracias al encuentro de dos mundos.

- Superaron a través de la música y los ambientes sonoros el estigma de que la Identidad Cultural está compuesta exclusivamente por los elementos tradicionales, reconociendo así la capacidad de la cultura para evolucionar y enriquecerse al compartir rasgos con otras culturas. E igualmente concluyeron cómo la memoria de un pueblo depende del contexto en el que se desarrolla y por tanto constituye un elemento de identidad que tiene significado sólo para los miembros de dicho contexto.

- Valoraron, a través de los saber-hacer medicinales y artesanales comunitarios, cómo dichos conocimientos heredados son igualmente válidos para satisfacer las propias necesidades de los pueblos y permitir su desarrollo, a la vez que es posible considerar la oportunidad de conjugar la tecnología y la tradición sin que eso implique un detrimento cultural, sino por el contrario un enriquecimiento producto de las interacciones actuales que reflejan una evolución cultural.

\section{Resultados}

La Gráfica 1 muestra el indicador principal emanado de la UNESCO (1982) para medir la variable de Identidad Cultural. En la prueba pre-test el 22\% del grupo experimental y el 32\% del grupo control (que fue el porcentaje más alto para este grupo) se colocó en el rango de mayor certeza; los valores inferiores en ambos grupos, con el 7\% y el 5\% respectivamente, reflejaron una certidumbre no del todo determinante; mientras que una certeza parcial obtuvo valores del 17\% en el grupo experimental y el 19\% en el control. En el grupo experimental predominó un poco convencimiento de la relevancia cultural con el 31\%, en comparación con el $28 \%$ de sus pares. Y finalmente el rango de menor valor respecto a dicha certeza obtuvo el $23 \%$ del grupo experimental y el 16\% del grupo control. 
Posteriormente, en la prueba pos-test los porcentajes más altos para ambas poblaciones los ocuparon el 38\% del grupo experimental y el 30\% del grupo control en el rango de mayor certeza, lo cual específicamente para el grupo experimental expuso un cambio drástico al pasar del 22 al 38\%; los valores inferiores en los dos grupos con el $8 \%$ para el experimental y el $6 \%$ para el de control reflejaron una certidumbre no del todo determinante, mientras que una certeza parcial obtuvo valores del $24 \%$ y $21 \%$ respectivamente. El poco convencimiento de la relevancia cultural se colocó con el 15\% del grupo experimental en comparación al 14\% del grupo control, y finalmente de manera correspondiente el rango de menor valor respecto a dicha certeza obtuvo el 15\% y 29\%.

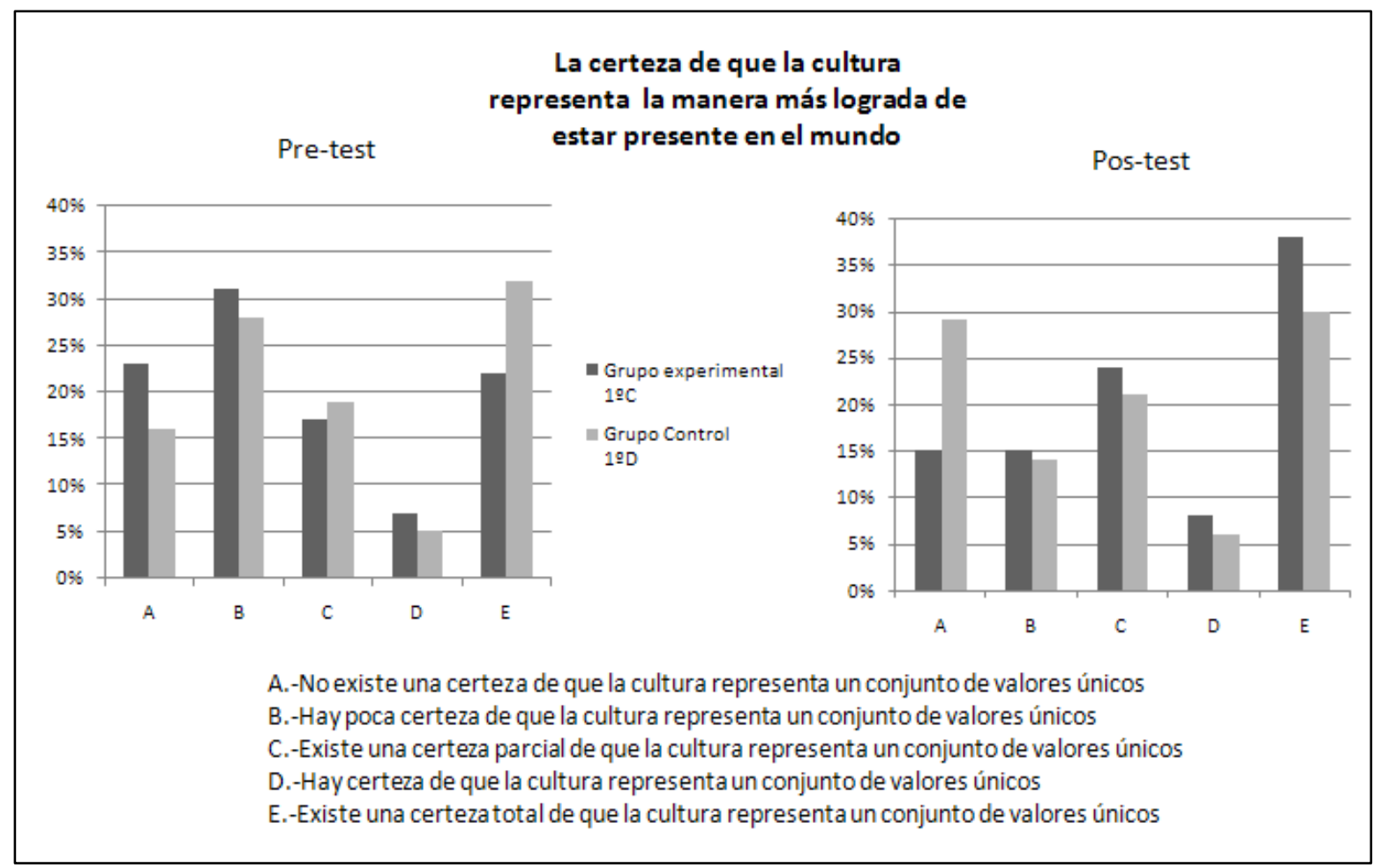

Gráfica 1. La certeza de que cada cultura representa un conjunto de valores único e irreemplazable.

Para estimar el grado de certeza de que la cultura representa un conjunto de valores único e irreemplazable, que sin embargo se ve enriquecida con los intercambios culturales, se pidió a los alumnos que expresaran sus juicios de valor respecto a la propia comunidad, que podrían poner de manifiesto, bien una tendencia a valorar una falta de personalidad cultural, un etnocentrismo cultural, o una sólida identidad. En ambos grupos predominó la valoración de la unicidad de la propia comunidad que sin embargo puede compartir rasgos con otras, con opiniones favorables de cerca de tres cuartas partes del grupo control en comparación a poco más de la mitad de la población en el experimental.

El reto planteado por los resultados de la prueba pre-test era promover actitudes que hicieran reconsiderar la opinión de ese $47 \%$ del grupo experimental que se colocaba entre el menosprecio y el etnocentrismo cultural. El éxito de las estrategias planteadas a partir de la prueba inicial, donde se puso mucha atención para evitar caer en la promoción de ideales etnocentristas, y al mismo tiempo diferenciar claramente una línea entre la identidad y el orgullo local, consistió en haber logrado que el 22\% de dichos alumnos recapacitara en relación al valor de la propia cultura. 
Uno de los ítems para medir este indicador pedía que se mencionaran ejemplos de manifestaciones culturales en las que hubiesen participado los estudiantes; sin embargo, lo realmente interesante en esta pregunta, más allá de las diferencias que exponen los resultados obtenidos, es el tipo de respuestas obtenidas, donde se ve reflejado el impacto de la intervención, de manera que en el pos-test fue posible identificar en el grupo experimental respuestas que abarcaban una concepción mucho más amplia de las expresiones culturales, al considerar elementos cotidianos como la lengua, la gastronomía y los rituales, y no sólo los eventos culturales como los desfiles, los festivales, y las fiestas cívicas de la localidad. Situación que se justifica al haber movilizado los conocimientos en la conceptualización y taxonomía del patrimonio cultural aportada por la UNESCO (1972): “El patrimonio cultural tiene dos vías de manifestación: la material, como edificaciones u objetos, y la inmaterial, como procesos, lenguas y técnicas".

Y de esta manera, al facilitar los conocimientos a los alumnos de tal forma que más que un ejercicio memorístico se motivara la reflexión, éstos fueron capaces de identificar con mayor facilidad tipos de manifestaciones y expresiones culturales, e incluso ampliar la concepción de los mismos.

Para la gráfica comparativa de la variable de Identidad Cultural (Gráfica 2) se utilizaron los resultados de los indicadores que sirvieron para medir "la defensa de las tradiciones, la historia y los valores morales, espirituales y éticos heredados de las generaciones pasadas" (UNESCO, 1982:6). La clara identificación durante el pre-test de elementos culturales que adscriben el sujeto a la comunidad fue mayor en el grupo control respecto al experimental (32 y 22\% respectivamente), mientras que la falta de reconocimiento hacia dichos elementos que diferencian de los demás grupos sociales, es decir, el polo negativo en la escala de valores, tuvo mayor incidencia en el $1^{\circ} \mathrm{D}(23 \%$ en contraposición al $15 \%$ del $1{ }^{\circ} \mathrm{C}$ ). En cambio, para el pos-test la clara identificación de elementos culturales que adscriben el sujeto a la comunidad aumentó considerablemente y fue mayor en el grupo experimental respecto al de control (39 contra 31\%), mientras que la falta de reconocimiento hacia dichos elementos que diferencian de los demás grupos sociales, es decir, el polo negativo en la escala de valores, tuvo esta vez mayor incidencia en el grupo control (27\% en contraposición al 15\% del experimental). 


\section{Identidad Cultural}
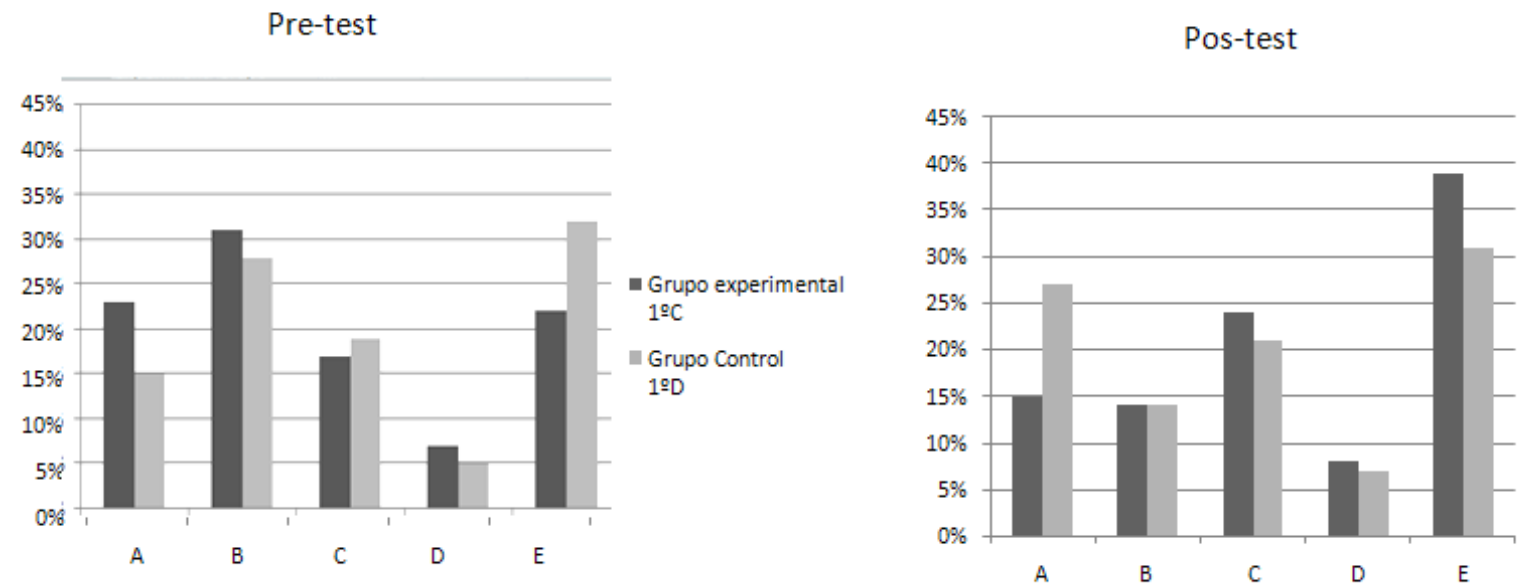

A.-El alumno no reconoce con claridad los elementos culturales que lo conformany que lo diferencian de los demás grupos B.-El alumno reconoce sólo algunos los elementos culturales que lo conforman y que lo diferencian de los demás grupos C.-El alumno reconoce parcialmente los elementos culturales que lo conforman y que lo diferencian de los demás grupos D.-El alumno reconoce muchos de los elementos culturales que lo conforman y que lo diferencian de los demás grupos E.-El alumno reconoce claramente los elementos culturales que lo conforman y que lo diferencian de los demás grupos

Gráfica 2. Comparativa de la variable identidad cultural.

Los datos aportados en dicha gráfica permiten vislumbrar con mayor facilidad una superioridad en el desarrollo de la Identidad Cultural del grupo experimental respecto al grupo control; puede observarse con mayor claridad el impacto y las variaciones presentes en el grupo experimental, que si bien continúa con la presencia de un cierto porcentaje de alumnos que no lograron reconocer con claridad los elementos culturales que lo conforman, sí reflejan resultados alentadores para un primer esfuerzo en cuanto al desarrollo de la auto-adscripción al grupo del que se forma parte.

\section{Conclusiones}

El contexto mexicano en cuanto a Educación Patrimonial se refiere, persigue el desarrollo de una Identidad Cultural a través de estrategias que involucran tan sólo el patrimonio material, recayendo así en la exaltación meramente de los bienes que son producto de una selección previa del Estado, y que en muchas ocasiones se encuentra fuera de la cotidianeidad del estudiante por tener éste su entorno en la periferia.

Partiendo de las aspiraciones en cuanto a la Educación Patrimonial que propone el marco normativo de la Educación Media Superior en México, es gracias a la metodología del modelo simbólico social que en este ejercicio se desarrollaron una serie de acciones conscientemente planificadas, donde sirviéndose del enfoque constructivista, se buscó lograr de manera óptima el proceso de enseñanza-aprendizaje para el fortalecimiento de una construcción de identidad cultural que permitiera desarrollar en el individuo lazos de pertenencia respecto al patrimonio.

La comparación de los resultados de una prueba pre-test y post-test en dos grupos del primer año de Educación Media Superior de una escuela ubicada en la periferia de la ciudad de Puebla, que sirvieron a manera de grupo control y grupo experimental, evidenciaron cómo las 
estrategias de aprendizaje que se sirven del Patrimonio Inmaterial contribuyen a reafirmar la Identidad Cultural, identificando y revalorizando elementos culturales presentes en la cotidianeidad que, si bien no se encuentran materializados, sí representan símbolos de identidad productos de un proceso histórico.

Las estrategias implementadas para promover la sensibilización a la herencia patrimonial permitió que la auto-percepción, como protagonista histórico construida parcialmente, se complementara y cambiara la tendencia en los alumnos a identificarse con los patrones de la globalidad para generar una visión más amplia en cuanto a manifestaciones culturales se refiere, revalorizando aquellos elementos que al estar tan presentes en su cotidiano suelen pasar inadvertidos. El reconocimiento de la presencia de elementos productos del devenir histórico en la propia identidad contribuyó como un primer paso para emprender en lo venidero acciones encaminadas al cuidado y preservación del patrimonio cultural.

El ejercicio evidenció que promover en el aula la motivación de actitudes que impliquen una sensibilización requiere de acciones más ambiciosas que busquen superar la mera cognición en los alumnos, la cual si bien es importante, resulta en un impacto que se ve mermado al carecer de actividades más vivenciales que favorezcan en el estudiante el desarrollo de un verdadero sentido de pertenencia.

La investigación expuso, por tanto, la necesidad de repensar estrategias de aprendizaje a partir del contexto del alumno, de tal manera que el docente sea capaz de abrir posibilidades de adaptación a las líneas propuestas institucionalmente. Lo que implica que, además de la consideración de las estructuras cognitivas presentes en el sujeto, es necesario reconocer que tanto la importancia como el compromiso del entorno son infaltables para favorecer la construcción del conocimiento en el individuo y por tanto deben replantearse estrategias a partir de tales premisas para promover el aprendizaje y el desarrollo de competencias, logrando una conciliación entre la secuencia programática de la asignatura y el contexto de los alumnos, para superar así la Dificultad Conceptual en la enseñanza patrimonial, es decir "la visión restrictiva (del término patrimonio) tanto en los materiales didácticos, como en la bibliografía especializada” (López, 2014, p.84).

La intervención didáctica que se sirve del patrimonio inmaterial, como la gastronomía, la música, la lengua, las creencias y los saberes heredados de generación en generación, permite superar los límites que una Educación Patrimonial basada meramente en la herencia patrimonial material, superando así el puente entre lo que históricamente les pertenece y la cotidianeidad.

\section{Referencias}

Asiáin, A. y Aznárez, M. (2012). Patrimonio Cultural Inmaterial y adquisición y desarrollo del lenguaje: tradición discursiva y psicodinámica oral. Huarte de San Juan. Filología y Didáctica de la Lengua, 12, 45-64.

Baumann, G. (2001). El enigma multicultural. Un replanteamiento de las identidades nacionales, étnicas y religiosas. Barcelona: Paidós

Bisbal, M. (2001). De cultura, comunicación y consumo cultural. Una misma perspectiva de análisis. Zer. Revista de estudios de comunicación, 12, 33-58.

De Allende, C.M. y Morones, G. (2006) Glosario de términos vinculados con la cooperación académica, ANUIES. Recuperado de: http:// www.anuies.mx/media/docs/convocatorias/pdf/glosariocoopnal2-jul06.pdf

Delors, J. (1996). La educación encierra un tesoro. Informe a la UNESCO de la Comisión Internacional sobre la Educación para el siglo XX. México: Ed. Dower

Fontal Merillas, O. (2003). La Educación Patrimonial. Teoría y práctica para el aula, el museo e Internet. 
Gijón: Ed. Trea S.L.

Fontal Merillas, O. y Marín Cepeda, S. (2011). Enfoques y Modelos de Educación Patrimonial en programas significativos de OEPE. Educación artística, Revista de investigación, 2, 91-96.

Freire, P. (2005). Pedagogía del oprimido. México : Ed. Siglo XXI.

Gabardón, J.F. (2005). La enseñanza del patrimonio. Propuestas educativas en torno al patrimonio local. Investigación en la Escuela, 56, 87-93.

Gil-Díez, I. (2013). La Educación Patrimonial en la Rioja. Berceo, 58, 77-88.

Gorosito, A.M. (1998). Identidad, cultura y nacionalidad. En Bayardo, R. y Lacarrieux, M. (comp.), 101-112. Globalización e Identidad Cultural. Buenos Aires: CICCUS,

Henríquez Sánchez, M.T. (2011). Lo que el ojo no ve. Políticas de lo inmaterial. Revista Atlántida: revista canaria de ciencias sociales, 3, 193-208.

Loison, M. (2008). Obstáculos epistemológicos, didácticos y pedagógicos para una enseñanza articulada de patrimonio. Iber: Didáctica de las ciencias sociales, geografía e historia, 58, 77-88.

López, I. (2014). La Educación Patrimonial. Análisis de tratamiento didáctico del patrimonio en los libros de Texto de CCSS en la Enseñanza Secundaria (Memoria de doctorado). Departamento de Didáctica de las Ciencias y Filosofía, Universidad de Huelva.

Martín Guglielmino, M. (2007). La difusión del patrimonio. Actualización y debate. Revista Electrónica de Patrimonio Histórico, 165, 151-162.

Moreno, A. (2012). World V alues Survey - México 2012. México: Instituto Tecnológico Autónomo de México.

Pinto, H. Y Molina, S. (2015) La Educación Patrimonial en los currículos de ciencias sociales en España y Portugal. Educatio Siglo XXI: Revista de la Facultad de Educación, 1, 103-128.

Sánchez Quintanar, A. (2002) Reencuentro con la Historia. Teoría y praxis de su enseñanza en México. Distrito Federal: FFyL, UNAM.

Secretaría de Educación Pública (2008). ACUERDO número 444 por el que se establecen las competencias que constituyen el marco curricular común del Sistema Nacional de Bachillerato. Recuperado de: http://www.ofmx.com.mx/documentos/pdf/Acuerdo444.pdf

United Nations Educational, Scientific and Cultural Organization (1972). Recomendación sobre la Protección, en el Ámbito Nacional, del Patrimonio Cultural y Natural. Recuperado de: http://portal.unesco.org/es/ev.phpURL_ID=13087\&URL_DO=DO_TOPIC\&URL_SEC TION=201.html

United Nations Educational, Scientific and Cultural Organization (1982). Declaración de México sobre las Políticas Culturales. Recuperado de: http:// portal.unesco.org/culture/es/files/35197/ 11919413801mexico_sp.pdf/ mexico_sp.pdf

United Nations Educational, Scientific and Cultural Organization (2003). Convención para la salvaguardia del patrimonio cultural inmaterial. Recuperado de http://portal.unesco.org/ es/ev.php URL_ID=17716\&URL_DO=DO_TOPIC\&URL_SECTION=201.html

Vizcaíno, F. (2005) Identidad nacional, sentido de pertenencia y autoadscripción étnica. En Béjar, R. (coord.) La identidad nacional mexicana como problema politico y cultural, 231-252. Nuevas miradas. Cuernavaca: UNAM, Centro Regional de Investigaciones Multidisciplinarias, 


\section{Sobre el autor}

Autor: Jonathan Almanza Ferrer

Institución: Escuela Técnica Superior de Turismo (Tuno - Campus Marseille, Francia)

Información biográfica profesional: Licenciado en Administración Turística, Maestro en Educación Superior. Líneas de investigación: Turismo, patrimonio y desarrollo, Educación Patrimonial.

Email: afjona@hotmail.com.

ORCID: no disponible

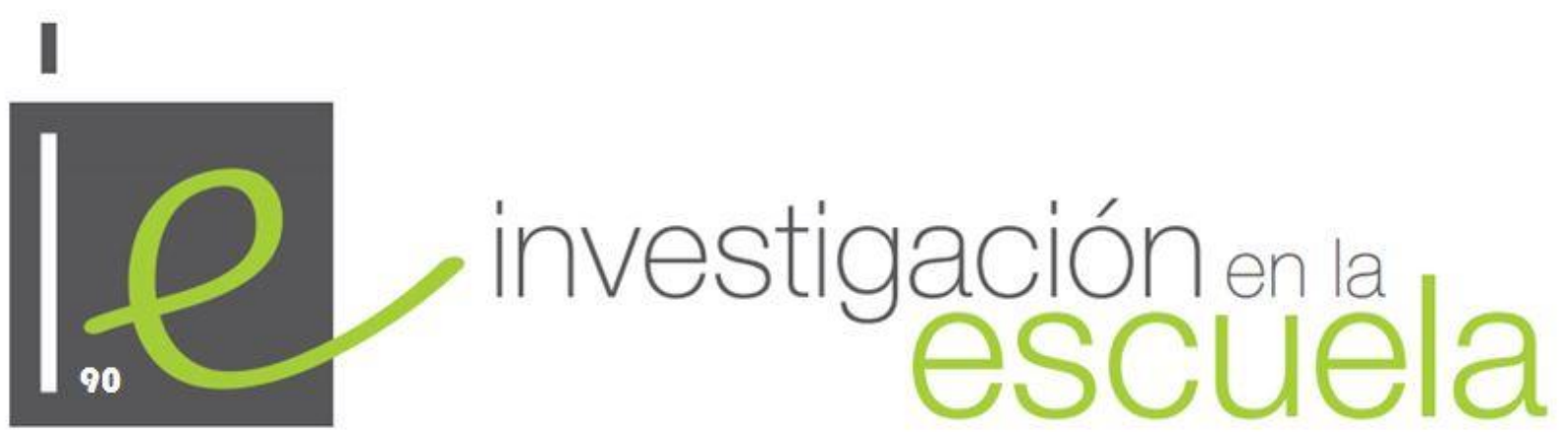

Revista académica evaluada por pares y de acceso abierto

ISSN 2443-9991

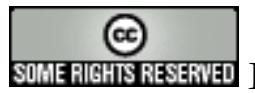
se de crédito y atribución al autor/es y a Investigación en la Escuela, se distribuya con propósitos no-comerciales, no se altere o transforme el trabajo original. Más detalles de la licencia de Creative Commons se encuentran en http://creativecommons.org/licenses/by-nc-sa/3.0 Cualquier otro uso debe ser aprobado en conjunto por el autor/es, o Investigación en la Escuela.

Contribuya con comentarios y sugerencias en la web de la revista. Por errores y sugerencias contacte a investigacionescuela@,ddcc.uhu.es 


\section{Investigación en la escuela}

Consejo de dirección: Ana Rivero García (Universidad de Sevilla), Nicolás de Alba Fernández (Universidad de Sevilla), Pedro Cañal de León (Universidad de Sevilla), Francisco F. García Pérez (Universidad de Sevilla), Gabriel Travé González, (Universidad de Huelva), Francisco F. Pozuelos Estrada (Universidad de Huelva)

Dirección: Ana Rivero García y Nicolás de Alba Fernández Técnico de edición: Francisco Javier López Sánchez

\section{Consejo editorial}

José Félix Angulo Rasco. Universidad de Cádiz Rosa Ma Ávila Ruiz. Universidad de Sevilla Pilar Azcárate Goded. Universidad de Cádiz Juan Bautista Martínez Rodríguez. Universidad de Granada

Nieves Blanco García. Universidad de Málaga Fernando Barragán Medero. Universidad de La Laguna José Carrillo Yáñez. Universidad de Huelva José Contreras Domingo. Universidad de Barcelona. Luis C. Contreras González. Universidad de Huelva Ana $\mathbf{M}^{\mathbf{a}}$ Criado García-Legaz. Universidad de Sevilla Rosario Cubero Pérez. Universidad de Sevilla José Ma Cuenca López. Universidad de Huelva Jesús Estepa Giménez. Universidad de Huelva Rafael Feito Alonso. Universidad Complutense (Madrid)

Francisco José García Gallardo. Universidad de Huelva

Soledad García Gómez. Universidad de Sevilla J. Eduardo García Díaz. Universidad de Sevilla
Fernando Hernández Hernández. Universidad de Barcelona

Salvador Llinares Ciscar. Universidad de Alicante Alfonso Luque Lozano. Universidad de Sevilla Rosa Martín del Pozo. Universidad Complutense (Madrid)

José Martín Toscano. IES Fernando Herrera (Sevilla) Jaume Martínez Bonafé. Universidad de Valencia F. Javier Merchán Iglesias. Universidad de Sevilla Emilia Moreno Sánchez. Universidad de Huelva. Rosario Ortega Ruiz. Universidad de Córdoba Antonio de Pro Bueno. Universidad de Murcia Fco. de Paula Rodríguez Miranda. Universidad de Huelva

Pedro Sáenz-López Buñuel. Universidad de Huelva Antoni Santisteban Fernández. Universidad Autónoma (Barcelona)

Emilio Solís Ramírez. Catedrático de IES. $\mathbf{M}^{\mathbf{a}}$ Victoria Sánchez García. Universidad de Sevilla. Magdalena Suárez Ortega. Universidad de Sevilla

\section{Consejo asesor}

Manuel Area Moreira. Universidad de La Laguna

Jaume Carbonell. Director Cuadernos de Pedagogía. Barcelona

César Coll. Universidad de Barcelona

Christopher Day. Universidad de Nothingham. U.K.

Juan Delval. Universidad Nacional de Educación a Distancia

John Elliott. Universidad de East Anglia. Norwich. U.K.

José Gimeno Sacritán. Universidad de Valencia

André Giordan. Universidad de Paris VII y Ginebra

Francisco Imbernón. Universidad de Barcelona

Ángel Pérez Gómez. Universidad de Málaga

Rafael Porlán Ariza. Universidad de Sevilla

Francesco Tonucci. Instituto de Pedagogía del C.N.R. Roma

Jurjo Torres Santomé. Universidad de A Coruña 
